\title{
The identification of nuclear av $\beta 3$ integrin in ovarian cancer: non-paradigmal localization with cancer promoting actions
}

\author{
Chen Seraya-Bareket $\mathbb{1}^{1,2}$, Avivit Weisz ${ }^{1,2}$, Elena Shinderman-Maman 1,2, Sharon Teper-Roth ${ }^{1,3}$, Dina Stamler ${ }^{1,3}$, \\ Nissim Arbib ${ }^{3,4}$, Yfat Kadan ${ }^{3,4}$, Ami Fishman ${ }^{3,4}$, Debora Kidron ${ }^{3,5}$, Evgeny Edelstein ${ }^{5}$, Martin Ellis ${ }^{1,3}$ and \\ Osnat Ashur-Fabian (1) ${ }^{1,2}$
}

\begin{abstract}
Nuclear translocation of transmembrane proteins was reported in high-grade serous ovarian cancer (HGSOC), a highly aggressive gynecological malignancy. Although the membrane receptor av $\beta 3$ integrin is amply expressed in HGSOC and involved in disease progression, its nuclear localization was never demonstrated. Nuclear av $\beta 3$ was explored in HGSOC cells (OVCAR3, KURAMOCHI, and JHOS4), nuclear localization signal (NLS) modified $\beta 3$ OVCAR3, Chinese hamster ovaries (CHO-K1) and human embryonic kidney (HEK293) before/after transfections with $\beta 3 / \beta 1$ integrins. We used the ImageStream technology, Western blots (WB), co immunoprecipitations (Co-IP), confocal immunofluorescence (IF) microscopy, flow cytometry for cell counts and cell cycle, wound healing assays and proteomics analyses. Fresh/archived tumor tissues were collected from nine HGSOC patients and normal ovarian and fallopian tube (FT) tissues from eight nononcological patients and assessed for nuclear av $\beta 3$ by WB, confocal IF microscopy and immunohistochemistry (IHC). We identified nuclear av $\beta 3$ in HGSOC cells and tissues, but not in normal ovaries and FTs. The nuclear integrin was Tyr 759 phosphorylated and functionally active. Nuclear av $\beta 3$ enriched OVCAR3 cells demonstrated induced proliferation and oncogenic signaling, intact colony formation ability and inhibited migration. Proteomics analyses revealed a network of nuclear av $\beta 3$-bound proteins, many of which with key cancer-relevant activities. Identification of atypical nuclear localization of the avß33 integrin in HGSOC challenges the prevalent conception that the setting in which this receptor exerts its pleiotropic actions is exclusively at the cell membrane. This discovery proposes av $\beta 3$ moonlighting functions and may improve our understanding of the molecular basis of ovarian cancer pathogenesis.
\end{abstract}

\section{Introduction}

High-grade serous ovarian cancer (HGSOC) is a gynecological malignancy that, although about one-tenth as common as breast cancer, is associated with a disproportionately large number of deaths due to late diagnosis after the disease has metastasized to distant sites ${ }^{1}$.

\footnotetext{
Correspondence: Osnat Ashur-Fabian (osnataf@gmail.com)

${ }^{1}$ Translational Oncology Laboratory, Hematology Institute and Blood Bank, Meir Medical Center, 44821 Kfar-Saba, Israel

${ }^{2}$ Department of Human Molecular Genetics and Biochemistry, Sackler Faculty of Medicine, Tel Aviv University, 6997801 Tel Aviv, Israel
}

Full list of author information is available at the end of the article
Recent studies strongly suggest that the fallopian tube (FT) epithelium is the source of HGSOC, rather than the ovarian surface epithelium as previously believed ${ }^{2}$. The first step is a mutation in FT cells in the tumor suppressor gene p53, known as "p53 signature", later additional mutations accumulate and the cells implant on the ovary, resulting in HGSOC tumor.

Emerging evidence has shown the translocation of transmembrane proteins from the cell membrane to the nucleus ${ }^{3}$. Such mislocalization of proteins has been suggested to alter the original protein activity and to be associated with several human diseases, including cancer ${ }^{4}$. 
Data on nuclear translocations of membrane receptors in HGSOC is limited to epidermal growth factor receptor (EGFR) and E cadherin ${ }^{5,6}$ and identification of additional nuclear receptor trafficking may lead to better understanding of disease pathogenesis. HGSOC metastasis is characterized by a spread of tumor cells inside the peritoneal cavity and their subsequent invasion within the organs of the pelvis ${ }^{1}$. These adhesion-related events are partly dependent on integrins, plasma membrane receptors with profound effects on cancer development ${ }^{7}$. Among the 24 integrins, $\alpha v \beta 3$ integrin is amply expressed on the membrane of HGSOC cells and participates in disease progression and invasion ${ }^{8-11}$. We describe here the first indication for an intranuclear localization of the $\alpha v \beta 3$ integrin, its interactions with an array of cancerrelevant proteins and a suggested role in HGSOC cell proliferation.

\section{Results}

\section{av $\beta 3$ integrin localizes in the nucleus of HGSOC cells}

To study the integrin localization we used an antibody that recognizes the $\alpha v \beta 3$ integrin dimeric form (clone LM609) and double stained the cells for membrane integrin in red and intracellular integrin in green. The cells were visualized using the ImageStream flow cytometer, an advanced technology acquiring both integrated fluorescence signals and high quality fluorescence images, combined with multiparametric cell analyses. This allowed us to identify atypical $\alpha v \beta 3$ nuclear localization in OVCAR3, KURAMOCHI, and JHOS4 (Fig. 1a), HGSOC cell lines which highly resemble the genetic profile of the human disease $\mathrm{e}^{12}$. Moreover, a similarity score, which measures the overlap between the pixel intensities of paired fluorescent channels in a single image, was calculated by regression analysis using the Pearson's correlation coefficient. A similarity score above a cutoff $>1.5$ (Supplementary Fig. 1A) confirmed positive correlation between the green (intracellular $\alpha v \beta 3$ ) and the blue (cell nucleus) channels in OVCAR3 and KURAMOCHI cells $(1.95 \pm 0.37$ and $1.95 \pm 0.34$, respectively) and no similarities between the remaining channels. Further verification was provided by WB analysis of cytosolic, membrane, and nuclear proteins. Anti- $\beta 3$ monomer antibody was used as a proxy for the integrin dimer $^{13}$, as in nonplatelet cells it complexes only with the $\alpha v$ monomer ${ }^{7}$. Comparable with the ImageStream results, $\beta 3$ integrin was identified, besides the membrane and cytosol, also within the nucleus of HGSOC cells (Fig. 1b). Notably, the nuclear integrin was negligible (Fig. 1c) in normal Chinese hamster ovary cells (CHO-K1), as well as in immortalized human fallopian tube cells (FT282, FT109, and FT237), considered the cell-of-origin of HGSOC $^{2}$. Fraction purity was confirmed for all models (Supplementary Fig. 1B-D) and normalized band quantifications in Supplementary Fig. 1E. Confocal microscopy images of a single $0.5 \mu \mathrm{m}$ optical section provided the final confirmation for a nuclear $\alpha v \beta 3$ in a subpopulation of OVCAR3 cells (Fig. 1d, left panel) using a functionally active $\alpha v \beta 3$ epitope antibody ${ }^{14}$. Notably, no nuclear integrin was observed in OVCAR3 cells during mitosis, as was shown before for another integrin member which resides underneath or close to the nucleus during cell division ${ }^{15}$. Similar nuclear localization was observed in KURAMOCHI and JHOS4 using an antibody for the integrin dimeric form (Fig. 1d, middle and right panels, respectively). Negative controls are depicted in Supplementary Fig. 1F. The staining pattern suggests integrin interchromatin clustering, probably in nuclear speckles.

Next, the existence of nuclear $\alpha v \beta 3$ integrin was explored in formalin-fixed paraffin-embedded (FFPE) tumors from nine HGSOC patients (Pt\#1-9) and ovarian

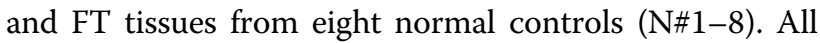
samples underwent analysis for $\mathrm{p} 53$, pax8, or $\mathrm{p} 16^{16}$, to confirm tumor/normal regions (Supplementary Fig. 2A, B, respectively). Fresh tissues were further collected from five HGSOC patients (Pt\#1-5) and two normal controls $(\mathrm{N} \# 1-2)$ and nuclear proteins were extracted. WB analyses confirmed the presence of variable levels of nuclear $\alpha v \beta 3$ in HGSOC tumors (Fig. 2a). Fraction purity is presented in Supplementary Fig. 2C and band quantifications in Supplementary Fig. 2D. A parallel immunohistochemistry (IHC) analyses, using an integrin dimer antibody, supported this observation. A representative IHC image displays characteristic nuclear p53 positive tumor cells (Fig. 2b, left panel) with scattered nuclear $\alpha v \beta 3$ staining (Fig. 2b, right panel). Additional patients are displayed in Supplementary Fig. 2E. For six tumors IF confocal microscopy images (Fig. 2c), demonstrating nuclear integrin in a subset of tumor cells. Notably, tumor-free (p53 negative) FT serous epithelia from HGSOC patient \#1 displayed insignificant integrin staining (Supplementary Fig. 2F). Similarly, lack of nuclear integrin was confirmed in nuclear extracts of normal FTs and ovarian tissues by WB (Fig. 2d) with background membranal $\alpha v \beta 3$ staining in the FT serous as well as the ovaries (Fig. 2e). The remaining normal FT samples and ovaries are presented in Supplementary Fig. 2G, H, respectively. Collectively, these results laid the basis that, besides the well-recognized membrane localization, there is a pool of nuclear $\alpha \mathrm{v} \beta 3$ in human HGSOC cells and tissues.

\section{av $\beta 3$ traffics to the nucleus in normal integrin-transfected cells}

We were next interested to explore nuclear $\alpha v \beta 3$ in normal cells forced to express this receptor. HEK293 cells that display endogenous $\alpha v$ but not $\beta 3$ ( $\alpha v \beta 3$ negative), were stably transfected with $\beta 3$ expression plasmid (HEK293 33 , $\alpha v \beta 3$ positive) or $\beta 1$ expression plasmid 

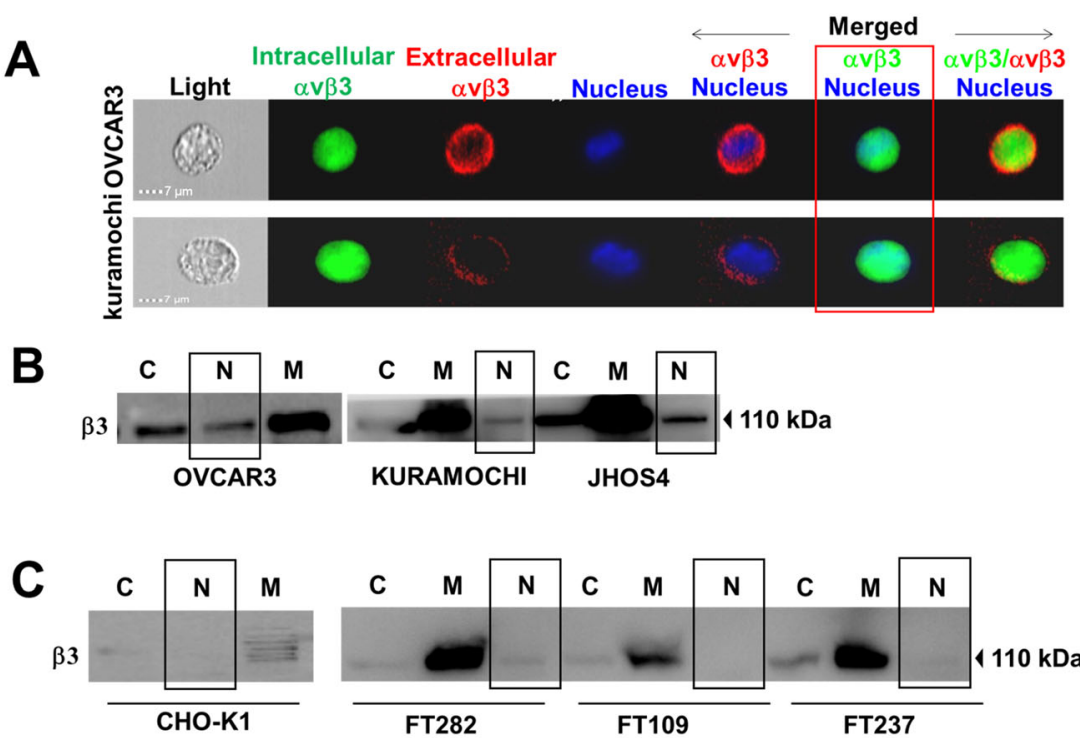

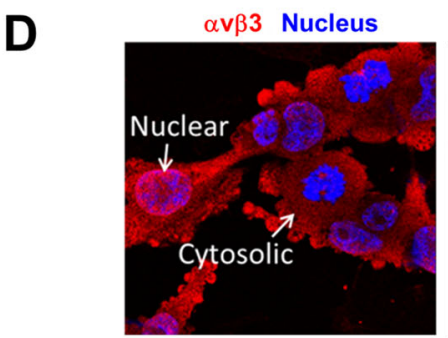

OVCAR3

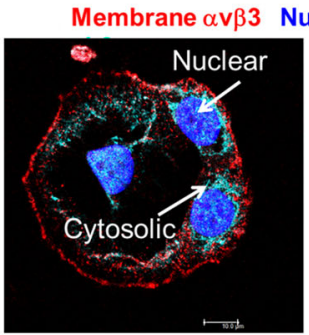

KURAMOCHI

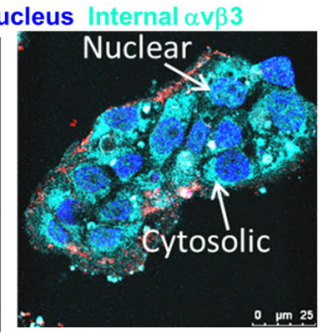

JHOS4

Fig. 1 Nuclear av $\beta 3$ in HGSOC cells. Extracellular and intracellular integrins were analyzed by a ImageStream of HGSOC cells $\left(1 \times 10^{5}\right)$ stained for membrane (red, PE-labeled) and intracellular avß3 (green, NL493-labeld). The nucleus was stained blue with Hoechst. 20x magnification images of separate and merged channels are shown. b, c Cellular protein fractions from $10 \times 10^{6} \mathrm{HGSOC}$ cells, normal ovaries (CHO-K1) and fallopian tube cell lines (FT282, FT109, and FT237) were extracted by Fraction PREP ${ }^{\text {TM }}$ Cell Fractionation kit. Proteins were separated using 10\% polyacrylamide gels, and analyzed by WB with antibodies against $\beta 3$. A representative blot of at least three independent repeats is shown. $C$ cytosolic fraction, $M$ membrane fraction, N nuclear fraction. Loading controls and fraction purity are presented in Supplementary Fig. 1B-D. d Confocal microscopy in OVCAR3 (active-avB3 in red, NL557), KURAMOCHI and JHOS4 (extracellular integrin in red, NL557; intracellular integrin in turquoise, BP680). In all images, a single $0.5 \mu \mathrm{m}$ optical section is presented using LeicaSP5 confocal microscopy, with a single cut images using $63 \times$ objective. Cells $\left(1 \times 10^{5}\right)$ were seeded in glass bottom 6-well plates. The nucleus was stained blue with Hoechst. Negative isotype lgG staining was validated (Supplementary Fig. 1F).

(HEK293 $\beta 1, \alpha v \beta 1$ positive/ $\alpha v \beta 3$ negative). Supplementary Fig. 3A depicts $\alpha v \beta 3$ membrane level in these cells. Next, equal amounts of cells were collected (Supplementary Fig. 3B) and cytosolic, nuclear, and membrane proteins were extracted. The nuclear fraction was devoid of GRP78, an ER protein extracted with the membrane proteins, excluding cross contamination between fractions (Supplementary Fig. 3C). Additional verification for fraction purity is provided in Supplementary Fig. 3D. WB analysis, using specific antibodies recognizing $\alpha v$ and $\beta 3$ monomers, confirmed nuclear integrin expression only in the $\beta 3$-transfected cells (Fig. 3a). Bands quantifications are depicted in Supplementary Fig. 3E. Using two antibodies that recognize active $\alpha \mathrm{v} \beta 3$ epitopes, CRC54 clone $^{14}$ or the pY759 phosphorylated form ${ }^{17}$, established that the nuclear receptor in the transfected cell model is phosphorylated and functionally active. ImageStream technology further confirmed the existence of nuclear integrin in HEK293ß3 (Fig. 3b), with a high similarity score between the green (intracellular $\alpha v \beta 3$ ) and the blue (cell nucleus) channels $(1.95 \pm 0.3)$, and no similarities between the remaining channels (Supplementary Fig. 3F). Lastly, IP with an anti $\alpha \mathrm{v} \beta 3$ antibody that recognizes the integrin dimer (clone LM609) established the presence of both integrin monomers in the nuclear fraction (Fig. 3c). IgG isotype (Supplementary Fig. 3G) and nontransfected cells (Supplementary Fig. 3H) served as negative controls.

\section{Enrichment of nuclear av $\beta 3$ integrin promotes HGSOC cell proliferation}

We next explored the functional role of the nuclear $\alpha v \beta 3$ in HGSOC. The possibility of mutating putative 

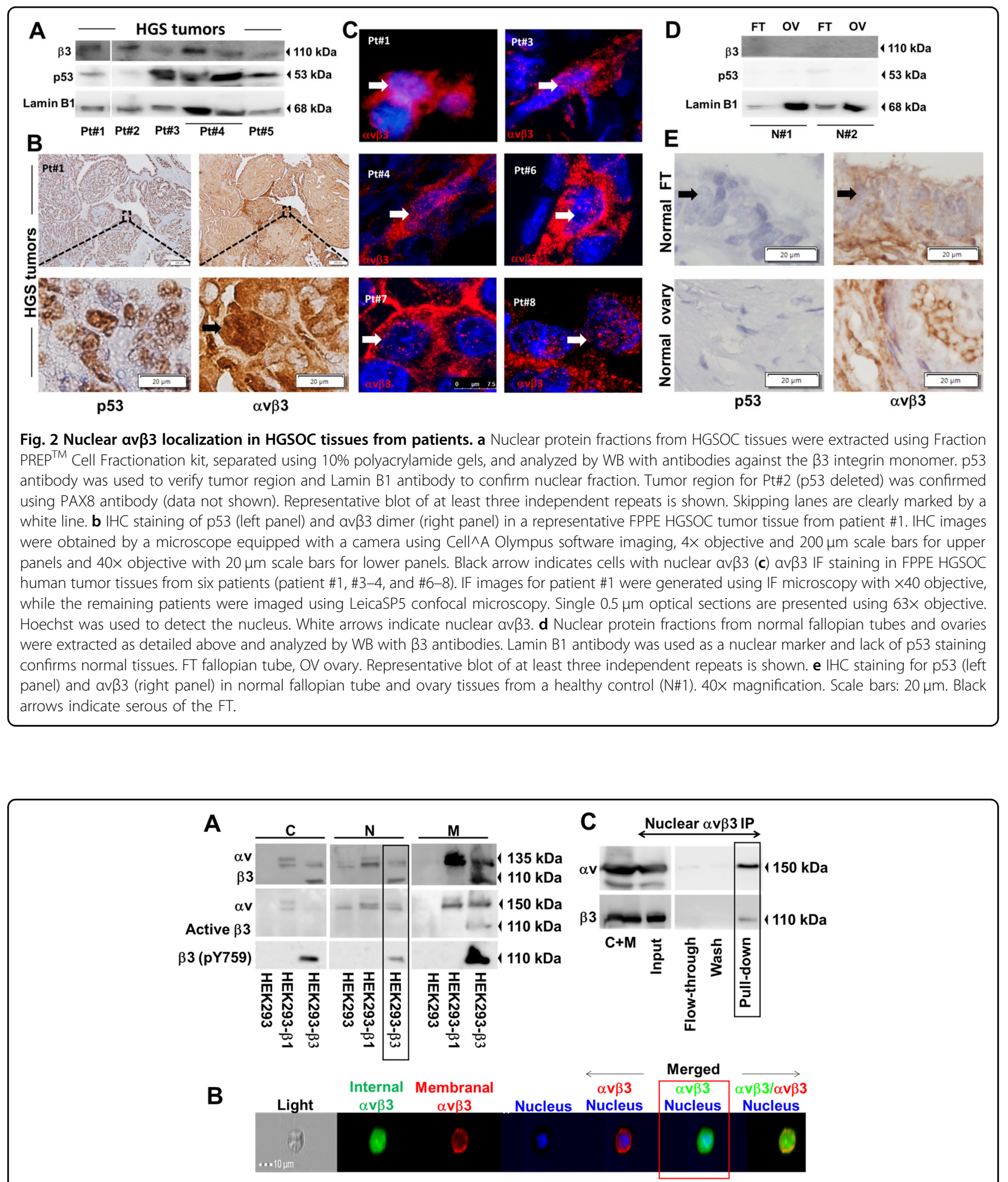

Fig. 3 Nuclear av $\beta 3$ localization in integrin transfected HEK293 cells. a WB analysis of av/ $\beta 3$ monomers, ligand occupied $\beta 3$ and phosphorylated $\beta 3$ in native and integrin transfected HEK293 cells. Fraction loading and purity is depicted in Supplementary Fig. 3B-D. b Extracellular and intracellular integrin was analyzed in av $\beta 3$-HEK293 cells by ImageStream. The cells $\left(1 \times 10^{5}\right)$ were stained for membrane (red, PE-labeled) and intracellular av $\beta 3$ (green, NL493-labeled). 20× magnification images of separate and merged channels are shown. c IP of nuclear av $\beta 3$-HEK 293 cells $\left(5 \times 10^{6}\right)$ using anti av $\beta 3$-antibody. Immunoblot with $\beta 3$ or av monomer antibodies is shown. Negative controls are depicted in Supplementary Fig. 3G-H. c cytosolic fraction, $\mathrm{M}$ membrane fraction, $\mathrm{N}$ nuclear fraction. Experiments are representative of two independent repeats. 


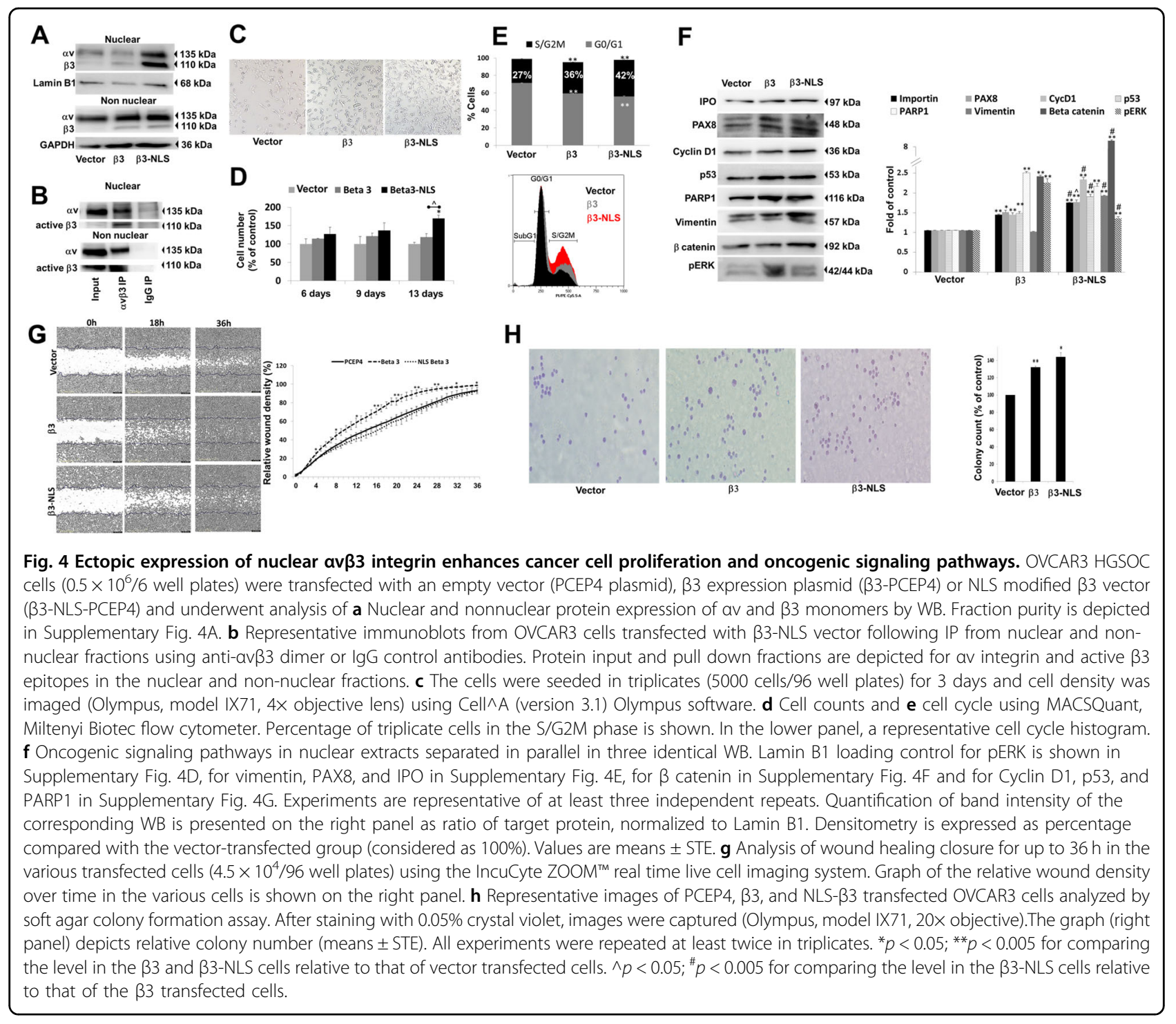

NLS motifs in the $\alpha v$ and $\beta 3$ monomers was excluded due to weak prediction scores, identified using bioinformatics tools (data not shown). Loss-of-function approach was also disqualified, as it would have significantly altered the membrane integrin expression and the cell phenotype. In addition, the relatively low amount of $\alpha v \beta 3$ in the nucleus makes it challenging to study the actual nuclear function and distinguish it from that of the membrane. To overcome these limitations, we set to ectopically enrich $\alpha v \beta 3$ amount inside the nucleus. For that, we modified a $\beta 3$ expression plasmid (PCEP4 backbone) with NLS motif at the $\mathrm{C}$ terminal end of the mRNA sequence ( $\beta 3$-NLSPCEP4). OVCAR3 cells, chosen as the proof of concept HGSOC model, were transfected with PCEP4, $\beta 3$-PCEP4, or $\beta 3$-NLS-PCEP4 vectors and stable lines were generated, following antibiotics selection. Enhanced nuclear integrin expression was confirmed in the OVCAR3- $\beta 3$ NLS cells (Fig. 4a, upper panel), compared to cells transfected with native $\beta 3$ or empty plasmids, while membrane integrin expressions were comparable between the transfected cells (Fig. 4a, lower panel). Fraction purity is depicted in Supplementary Fig. 4A. Moreover, by performing IP, the NLS-modified $\beta 3$ vector was shown to associate with the native $\alpha \mathrm{v}$ monomer and to present an active $\beta 3$ integrin conformation in both membrane and nuclear fractions in the transfected cells (Fig. 4b). In contrast, actin and talin, two cytoskeletal proteins which may physically connect between the membrane integrin and the nuclear lamina, were not bound to the $\alpha v \beta 3$ in the nuclear fractions of OVCAR3$\beta 3-N L S$ cells and HEK293b3 transfected cells (Supplementary Fig. 4B). This suggests that the identification of 
the nuclear integrin may not be attributed to cytosolic chemical annexation. Therefore, this system is a valid platform for deciphering the potential contribution of the nuclear $\alpha \mathrm{v} \beta 3$ in HGSOC.

First, the various transfected cells were equally seeded in triplicates and assessed after 3 days by microscopy. Results showed significantly higher cell density in OVCAR3 cells enriched with the nuclear $\alpha v \beta 3$ compared to cells transfected with an empty vector or the native integrin (Fig. 4c). Continuous growth for up to 13 days confirmed this phenotype by flow cytometry analyses of absolute cell counts (Fig. 4d), with a 1.67-fold increase at study end in the NLS-integrin transfected cells compared to empty and $\beta 3$ vectors $(p<0.005)$. A similar trend in the percentage of cells undergoing DNA synthesis and mitosis (S/G2M phase) was demonstrated by cell cycle analysis in the $\beta 3$-NLS transfected cells (Fig. 4e). A representative cell cycle histogram is shown in the lower panel. Next, nuclear proteins were extracted from the various transfected cells. Fraction loadings are presented in Supplementary Fig. 4C-F. Representative Western blots of an array of signaling pathways are shown in Fig. 4f and quantifications is shown on the right panel. Relative to empty-vector transfected cells, an increase in importin beta (IPO), involved in cargo delivery into the nucleus, was shown in $\beta 3$-transfected cells (1.3-fold, $p<0.005)$ and more so in $\beta 3$-NLS cells $(1.66$-fold, $p<0.005)$. Similarly, higher level of PAX8 and cyclin D1, both involved in cell proliferation, were shown in $\beta 3$ overexpressing cells (1.4fold and 1.37-fold, respectively). This effect was significantly more pronounced in the $\beta 3$-NLS cells with a 1.67-fold increase for PAX8 and a 2.2-fold increase for cyclin D1 $(p<0.005)$. Moreover, the level of OVCAR3 gain-of-function p53 mutant (R248Q), was induced in the $\beta 3$-transfected cells by 1.4 -fold and more potently in the $\beta 3$-NLS transfected cells $(1.8$-fold, $p<0.005)$. Nuclear vimentin and $\beta$ catenin, both associated with epithelial to mesenchymal transition (EMT), were also significantly induced in the $\beta 3$-NLS cells (1.8-fold and 8.3-fold, respectively, $p<0.005)$, compared to cells transfected with the $\beta 3$ vector ( 0.96 -fold and 2.3 -fold respectively). In contrast, the $\beta 3$-tranfected cells displayed greater induction in PARP1 (2.4-fold) and phosphorylated ERK (pERK, 2.2-fold), compared to the 33 -NLS cells (2-fold and 1.2fold, respectively, $p<0.005$ ), suggesting that this signaling is primarily regulated via the membrane integrin. The contribution of the nuclear integrin to ovarian cancer cell migration was next assessed by wound-healing assay (Fig. $4 \mathrm{~g})$. The relative wound density is depicted on the right panel. While $\beta 3$ accelerated wound healing closure, the NLS- $\beta 3$ cells displayed rate comparable with that of the control cells, suggesting, besides dominance of the membrane integrin, that the nuclear integrin may inhibit cell migration. Nonetheless, soft agar colony formation
(Fig. 4h) remained intact in cells expressing the NLSmodified integrin, signifying that nuclear enrichment did not hinder the tumorigenic potential of the malignant cells.

\section{The nuclear av $\beta 3$ complexes with central cancer-relevant proteins}

Finally, we aimed to elucidate the biological function of the nuclear integrin through identification of potential interacting proteins. To that end, we performed $\alpha v \beta 3$ CoIP for nuclear extracts of cells overexpressing the integrin (HEK293ß3) and the three HGSOC cell lines (OVCAR3, JHOS4, and KURAMOCHI). The integrin dimer was pulled-down using $\alpha v \beta 3$ antibody (clone LM609) by magnetic beads. Co-IP performed without the primary antibody (beads only) or in integrin negative cells (HEK293), served as negative controls and was used to quantify the assay background. Nuclear proteins were analyzed by LC-MS/MS and considered nuclear integrinassociated (unique) when having greater than 4-fold binding from Co-IP with beads and complete absence in integrin negative cells.

Proteomics analysis identified a total of $843 \alpha v \beta 3$ associated proteins, of which approximately 514 in HEK293ß3 cells, 111 in OVCAR3, 169 in JHOS4 and 196 in KURAMOCHI (Supplementary Table 1). Notably, the majority of proteins displayed complete lack of assay background, providing high level of confidence in results validity. Interaction network between the proteins in the various cells (Supplementary Fig. 5), generated using the STRING tool ${ }^{18}$, confirmed protein-protein interaction (PPI) enrichment $p$-values of $<1.0 \mathrm{e}-16$ for HEK293 $\beta 3$ and JHOS4, $<5.3 \mathrm{e}-14$ for OVCAR3, and $<3.3 \mathrm{e}-10$ for KURAMOCHI. This indicates that the protein network has significantly more interactions among themselves and is biologically connected as a group, than what would be expected for a random set of proteins of similar size drawn from the genome. Using the PANTHER tool ${ }^{19}$ main subcategories of the nuclear $\alpha v \beta 3$-bound proteins are presented in pie charts (Fig. 5a). Biological processes mainly involve metabolic, cellular and biological regulation ( $27 \%, 32 \%$, and $17 \%$, respectively), molecular function is largely via protein binding (43\%) and catalytic activity $(31 \%)$, while protein class is commonly of nucleic acid binders (26\%) and transcription factors (11\%). Multiple signaling pathways were associated with the nuclear integrin, including p53 (10\%) and Wnt (4\%). Using Venny diagram tool $^{20}$, we assessed the shared versus unique proteins in the different cells (Fig. 5b). While HEK293 33 displayed only $16 \%$ similarity with the HGSOC protein panel, in the HGSOC cells between $33-50 \%$ of the proteins were shared with at least one other cell model. Statistical analyses of the proteomics data indicated significant difference between the three HGSOC cells $(p<$ 0.001 ), and more profoundly between these cells and 


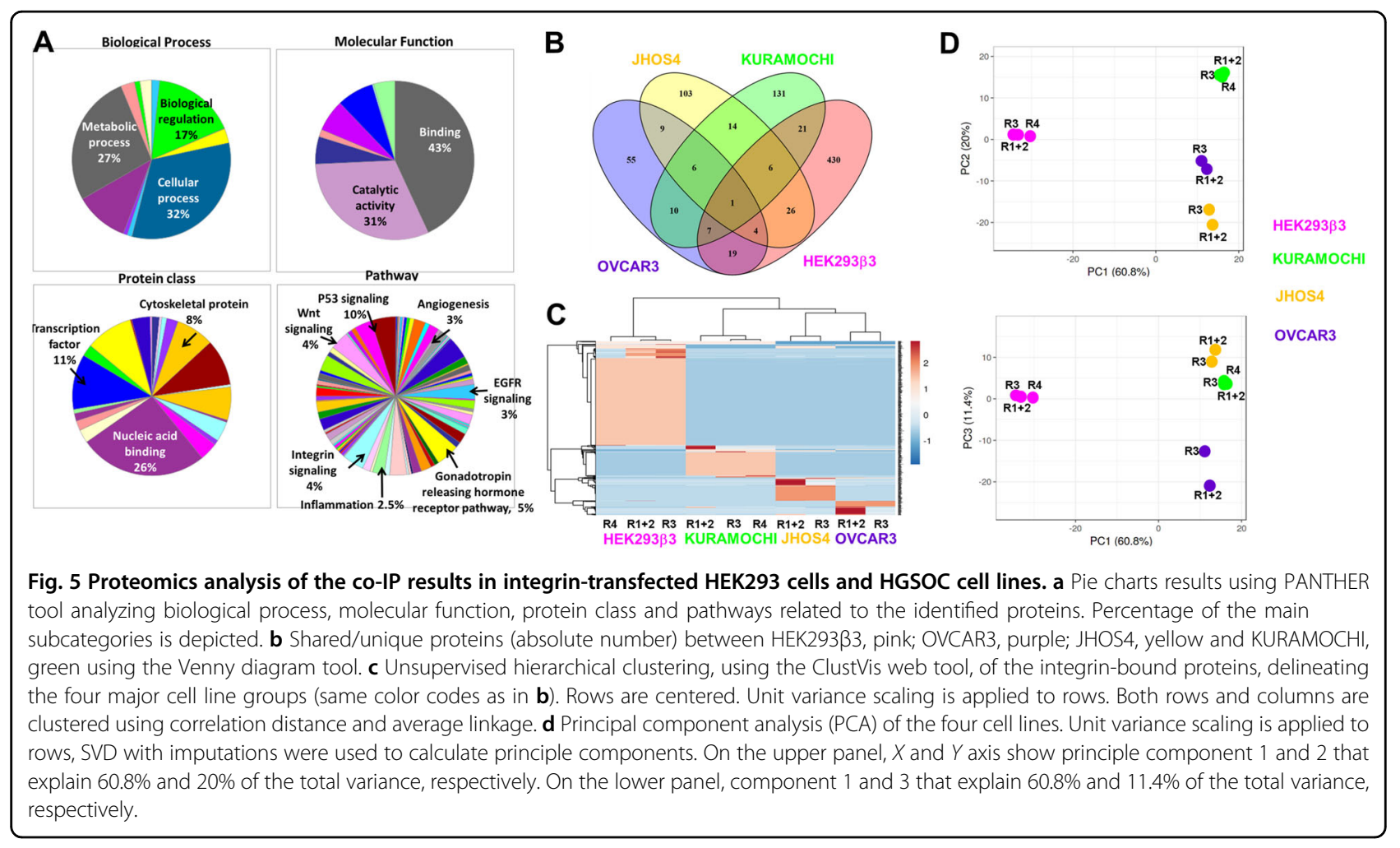

HEK293ß3 $\left(p<2.7 \times 10^{-22}\right)$. For visualizing clustering of multivariate data, the ClustVis web tool was used ${ }^{21}$. Heatmap results of unsupervised hierarchical clustering on the basis of the relative expression of the integrinbound proteins in the four cell groups, clearly delineated that the HGSOC cells cluster closer compared to the integrin transfected normal cells (Fig. 5c). Principal component analysis (PCA) further confirmed the segregation of the HGSOC clusters from that of the HEK29333 cells (Fig. 5d). This corresponds with the mutual disease origin shared by the HGSOC lines. Notably, in agreement with previous published works ${ }^{12}$, both analyses suggested a closer similarity between JHOS4 and KURAMOCHI compared to OVCAR3.

Lastly, we focused on 57 integrin-bound proteins that were shared between the various HGSOC cells (Table 1). Seventy-seven percent of these proteins were present in KURAMOCHI and $67 \%$ in JHOS4 and OVCAR3. In contrast, only $30 \%$ of these proteins were eluted with the nuclear integrin in HEK29333, although these cells express significantly higher levels of $\alpha v \beta 3$ and display superior number of integrin-bound proteins. This further accentuates the distinction observed between HEK29333 and the HGSOC cells panel using cluster analysis methods. According to the gene ontology (GO), the shared proteins belong to ten categories of biological processes. These include eight proteins involved in cell cycle and mitosis, among which Cullin-5 (CUL5) was the only protein that was commonly eluted in both the transfected cells and the entire HGSOC panel. We also identified proteins associated with apoptosis, such as CCAR1 and RMDN3, only in the HGSOC cell models. Notably, the nuclear integrin was bound to proteins known to be complexed with the membrane integrin ${ }^{22}$, including the cytoskeletal proteins Filamins (FLNA and FLNC), palladin (PALLD), and RAS-GTPase-activating-like protein (IQGAP1). Similarly, integrin linked kinase (ILK) and Talin 1 (TLN1) were identified, although only in specific cell models. Collectively, this indicates that at least some of these canonical proteins also interact with $\alpha v \beta 3$ within the nuclear compartment. In addition, a large group of proteins regulating both transcription and translation were associated with the nuclear $\alpha v \beta 3$, including the integrator complex subunit 2 (INTS2) and the eukaryotic translation initiation factor 5B (EIF5B). Lastly, several proteins involved in RNA, vesicles and protein transport, were identified, for example the translocation protein SEC62. Additional proteins facilitating in-and-out nuclear trafficking, including exportin, importins, clathrins, and nexins were also integrin bound, although unique subunits were identified in the various cell models. This, combined with the observed importin induction in the NLS-modified integrin cells, proposes a trafficking mechanism for the nuclear integrin. Collectively, the nuclear $\alpha v \beta 3$ interactome suggests potentially novel moonlighting activities for this receptor. 
Table 1 Shared nuclear av $\beta 3$-integrin bound proteins from in the various cell models.

\begin{tabular}{|c|c|c|c|c|c|c|c|}
\hline \multirow{2}{*}{ Biological process (GO) } & \multirow{2}{*}{ Protein } & \multirow[b]{2}{*}{ Full name } & \multirow[b]{2}{*}{ Subcellular location } & \multicolumn{4}{|c|}{ Cell models } \\
\hline & & & & $293 \beta 3$ & OVCAR3 & JHOS4 & KURAMOCHI \\
\hline \multirow{8}{*}{ Cell cycle and mitosis } & CUL5 & Cullin-5 & Cytosol & & & & \\
\hline & NCAPH & Condensin complex subunit 2 & Nucleus & & & & \\
\hline & NCAPD2 & Condensin complex subunit 1 & Nucleus & & & & \\
\hline & STK10 & Serine/threonine-protein kinase 10 & Membrane & & & & \\
\hline & TPR & Nucleoprotein TP & Nucleus & & & & \\
\hline & ZNF830 & Zinc finger protein 830 & Nucleus & & & & \\
\hline & CLTC & Clathrin heavy chain 1 & Cytoskeleton & & & & \\
\hline & FAM120A & Constitutive coactivator of PPAR-gamma-like protein 1 & Membrane & & & & \\
\hline \multirow{4}{*}{ Apoptotic process } & RMDN3 & Regulator of microtubule dynamics protein 3 & Nucleus, mitochondrion & & & & \\
\hline & CCAR1 & Cell division cycle and apoptosis regulator protein 1 & Cytoplasm & & & & \\
\hline & MAP1S & Microtubule-associated protein $1 \mathrm{~S}$ & Nucleus, cytoskeleton, cytosol & & & & \\
\hline & NAA35 & $\mathrm{N}$-alpha-acetyltransferase 35 & Cytoplasm & & & & \\
\hline \multirow{2}{*}{ Differentiation } & PRRC2C & Proline-rich and coiled-coil-containing protein $2 \mathrm{C}$ & Cytosol, membrane & & & & \\
\hline & YTHDF2 & YTH domain-containing family protein 2 & Nucleus and cytosol & & & & \\
\hline \multirow{2}{*}{ DNA damage/repair } & TP53BP1 & TP53-binding protein 1 & Nucleus & & & & \\
\hline & FANCl & Fanconi anemia group I protein & Nucleus & & & & \\
\hline \multirow{2}{*}{ Metabolic process } & MTHFD1L & Monofunctional C1-tetrahydrofolate synthase & Mitochondrion & & & & \\
\hline & HDLBP & High density lipoprotein binding protein (Vigilin) & Nucleus & & & & \\
\hline \multirow{2}{*}{ Adhesion } & PRKCA & Protein kinase $\mathrm{C}$ alpha type & Nucleus, mitochondrion & & & & \\
\hline & IQGAP1 & Ras GTPase-activating-like protein IQGAP1 & Nucleus, membrane, cytoplasm & & & & \\
\hline \multirow{5}{*}{ Actin binding } & FLNA & Filamin A & Cytoskeleton & & & & \\
\hline & FLNC & Filamin C & Cytoskeleton & & & & \\
\hline & PLEC & Plectin & Cytoskeleton & & & & \\
\hline & MYLK & Myosin light chain kinase, smooth muscle & Cytoskeleton, cytoplasm & & & & \\
\hline & PALLD & Palladin & Cytoskeleton, focal adhesion & & & & \\
\hline \multirow{14}{*}{ Transcription regulation } & INTS2 & Integrator complex subunit 2 & Nucleus & & & & \\
\hline & ZNF516 & Zinc finger protein 516 & Nucleus & & & & \\
\hline & MED14 & Mediator of RNA polymerase II transcription subunit 14 & Nucleus & & & & \\
\hline & TAF6L & TAF6-like RNA polymerase II p300 & Nucleus & & & & \\
\hline & WAC & WW domain-containing adapter protein with coiled-coil & Nucleus & & & & \\
\hline & INTS14 & Integrator complex subunit 14 & Nucleus & & & & \\
\hline & FLIII & Protein flightless-1 homolog & Nucleus & & & & \\
\hline & FOXP1 & Cyclin-dependent kinase 1 & Nucleus & & & & \\
\hline & AKAP8L & A-kinase anchor protein 8-like & Nucleus & & & & \\
\hline & CREB1 & Cyclic AMP-responsive element-binding protein 1 & Nucleus & & & & \\
\hline & MYADM & Myeloid-associated differentiation marker & Membrane & & & & \\
\hline & RBM3 & RNA-binding protein 3 & Nucleus & & & & \\
\hline & RBM12B & RNA-binding protein 12B & Nucleoplasm & & & & \\
\hline & SIX1 & Homeobox protein SIX1 & Nucleus & & & & \\
\hline \multirow{10}{*}{ Translation regulation } & EIF5B & Eukaryotic translation initiation factor $5 \mathrm{~B}$ & Cytoplasm & & & & \\
\hline & GIGYF2 & GRB10-interacting GYF protein 2 & Cytosol, ER, golgi, endosome & & & & \\
\hline & CAND1 & Cullin-associated NEDD8-dissociated protein 1 & Nucleus & & & & \\
\hline & CARS & Cysteine--tRNA ligase & Cytoplasm & & & & \\
\hline & LSM14B & Protein LSM14 homolog B & Intracellular & & & & \\
\hline & RPL35A & $60 \mathrm{~S}$ ribosomal protein $\mathrm{L} 35 \mathrm{a}$ & Cytosol & & & & \\
\hline & RPS16 & 40 S ribosomal protein S16 & Nucleus, cytosol and exosome & & & & \\
\hline & NOSIP & Nitric oxide synthase-interacting protein & Nucleus & & & & \\
\hline & QARS & Glutamine--tRNA ligase & Cytosol, cytoplasm & & & & \\
\hline & USP8 & Ubiquitin carboxyl-terminal hydrolase 8 & Nucleus, endosome & & & & \\
\hline \multirow{8}{*}{ Transport } & SNRPD1 & Small nuclear ribonucleoprotein Sm D1 & Nucleus, Cytosol & & & & \\
\hline & SRSF11 & Serine/arginine-rich-splicing factor 11 & Nucleus & & & & \\
\hline & DHX36 & ATP-dependent DNA/RNA helicase DHX36 & Nucleus, cytosol, mitochondrion & & & & \\
\hline & SEC62 & Translocation protein SEC62 & ER & & & & \\
\hline & ESYT2 & Extended synaptotagmin-2 & ER & & & & \\
\hline & MTCH1 & Mitochondrial carrier homolog 1 & Mitochondrion & & & & \\
\hline & $\begin{array}{ll}\text { LETM1 } \\
\end{array}$ & Mitochondrial proton/calcium exchanger protein & Mitochondrion & & & & \\
\hline & NSF & Vesicle-fusing ATPase & Cytoplasm & & & & \\
\hline
\end{tabular}

The table depicts different categories of biological processes according to Gene Ontology (GO), the protein short and full names, subcellular location and absence (white color) or presence (gray color) in the various cells.

\section{Discussion}

The presence of cell surface receptors in the nucleus was recognized decades ago, however, this research field is still relatively neglected in cancer in general and ovarian cancer in particular. Although integrins are known to recycle to and from the plasma membrane ${ }^{23}$, work on nuclear integrin trafficking is scarce. Two reports suggested nuclear trafficking of the $\alpha v$ or $\beta 4$ integrin monomers in cancer cells $^{24,25}$. This trafficking, however, did not involve the full receptor form and was evident only following specific stimuli. In this work, we identified atypical nuclear localization of the full $\alpha v \beta 3$ integrin receptor in HGSOC cells, but not normal FT cells and tissues. Since mutated FT epithelium is recognized as the source of HGS carcinoma ${ }^{2}$, we postulate that $\alpha v \beta 3$ trafficking into the nucleus may take part in this transformation. The nuclear $\alpha \mathrm{v} \beta 3$ was phosphorylated in cells ectopically expressing the integrin, suggestive of an active receptor conformation ${ }^{17}$. Identification of a nuclear $\alpha v \beta 3$ reservoir challenges the prevalent conception that the setting in which this receptor exerts its pleiotropic actions is exclusively at the cell membrane and proposes protein moonlighting functions.

To lay a clear foundation for the functional role of the nuclear $\alpha v \beta 3$, we generated, using NLS-modified $\beta 3$ 
integrin vector, HGSOC cells with stably enhanced nuclear integrin expression. This cell platform established, by multiple complementary methods, that the nuclear $\alpha v \beta 3$ increased cancer cell proliferation and activation of a host of oncogenic proteins. Among which, elevation in the gain-of-function R248Q mutant $\mathrm{p} 53^{26}$, cyclin D1 and $\beta$ catenin, known to transcriptionally regulate cyclin $D 1^{27}$, as well as vimentin which like $\beta$ catenin is involved in EMT and ovarian cancer progression ${ }^{28}$. PAX8, a lineage marker of the fallopian tube, shown to be associated with enhanced ovarian cancer proliferation ${ }^{29}$, was also induced. In addition, NLS-modified integrin cells exhibited induction in importin beta, a nuclear import $\operatorname{protein}^{30}$, signifying a mode of nuclear delivery. Our results are in agreement with reports on oncogenic pathway activation by retrograde nuclear trafficking of other transmembrane proteins ${ }^{4}$. In contrast, activation of ERK and cell migration were observed only in cells expressing the native $\alpha v \beta 3$, suggesting regulation primarily via the membrane integrin, roles that are wellestablished ${ }^{31,32}$. Collectively, although membrane integrins are known to govern signal transduction pathways ${ }^{22}$ and nuclear alterations ${ }^{33}$, our current work accentuates the critical novel role these receptors may play directly from within the nuclear compartment.

Using proteomics analysis, a network of $\alpha v \beta 3$-interacting proteins was revealed in the various cell models. Notably, a significantly higher number of proteins were identified in the integrin-transfected cells, suggesting that this platform facilitates identification. HGSOC cells and $\alpha \mathrm{v} \beta 3$-transfected normal cells distinctly cluster, proposing that the protein complexes are cell type and/ or disease-related. Among the nuclear-integrincomplexed proteins, we identified a group involved in cell cycle and mitosis. These include CUL5, a G2/M blockade protein that is downregulated in many tumor types, including ovarian cancer ${ }^{34}$, resulting in substantial proliferation. Interestingly, this protein was shown to target $\beta 1$, another integrin family member, for degradation $^{35}$ and to regulate focal adhesion dynamics in epithelial cells ${ }^{36}$. It remains to be studied whether complexing of CUL5 with the nuclear $\alpha \mathrm{v} \beta 3$ participates in its turnover and tumor-promoting actions. Several proteins involved in apoptosis were also nuclear integrin-bound, including CCAR1, a key regulator of chemotherapy-induced apoptosis ${ }^{37}$. CCAR1 correlates with ovarian cancer progression-free-survival ${ }^{38}$ and interacts with the nuclear estrogen receptor $\alpha$, an ovarian cancer relevant growth hormone, as well as with $\mathrm{SRC}^{39}$, which canonically complexes with the membrane $\alpha v \beta 3$ integrin. The second apoptotic-related protein, RMDN3, also known as PTPIP51, is highly expressed in several cancers and regulates and interconnects with central oncogenic signaling pathways ${ }^{40}$. Our work is the first to implicate a role for both proteins in complexing with the nuclear integrin in HGSOC.

Furthermore, we have unexpectedly identified proteins that commonly complex with the membrane $\alpha v \beta 3^{22}$, such as Filamins, Palladin, and Plectin, to be physically bound with this receptor inside the nucleus. Similarly, ILK, known to take part in focal adhesions ${ }^{41}$ was observed, although only in the integrin transfected cells. Nuclear trafficking of these proteins was previously reported in several malignancies ${ }^{42,43}$, providing support for our findings. Collectively, this suggests that nuclear translocation of integrin adherent structures may mediate their intranuclear effects, either through classical functions or via new mechanisms. We also identified key proteins involved in transcription and translation regulation bound with the nuclear integrin. These include INTS2, which is highly mutated in $\mathrm{HGSOC}^{44}$ and EIF5B, which is a critical regulatory pro survival node in several aggressive cancers ${ }^{45}$. Another central regulator of transcription, the Histone acetyltransferase p300 protein (EP300), was bound to the nuclear $\alpha v \beta 3$ in the integrin transfected cells and one of the HGSOC cell models. This specific protein was reported before in OVCAR3 cells to complex, following thyroid hormone stimulation, with the nuclear $\alpha v$ monomeric form, but not with the full receptor as observed by us $^{24}$. Lastly, several proteins involved in RNA, vesicles and protein transport were identified with the nuclear integrin. For example, the translocation protein SEC62, which is located at frequently amplified chromosomal region in numerous human cancers, including ovarian $^{46}$. With regards to transport, several proteins facilitating in-and-out nuclear trafficking, including exportin, importins, clathrins, and nexins ${ }^{4,30}$ were nuclear integrin bound. Specifically, importins and exportins act by binding the cargo protein via nuclear localization signal (NLS) motif ${ }^{47}$ and signify a potential trafficking mechanism for the nuclear integrin.

To conclude, we have identified a nuclear $\alpha v \beta 3$ integrin reservoir in ovarian cancer with a proposed role and interactions that may be distinct from its plasma membrane actions. This unique biological mechanism, which has been largely overlooked, adds to the multifaceted activities reported for $\alpha \mathrm{v} \beta 3$ and may lead to improved understanding of the molecular basis of ovarian cancer with a potential impact on other malignancies.

\section{Materials/Subjects and methods Antibodies}

$\alpha v \beta 3$ dimer (clone LM-609), $\alpha v$ monomer, $\mathrm{Na}^{+} \mathrm{K}^{+}$ ATPase $\alpha-1$ (Merck Millipore, Darmstadt, Germany); $\beta 3$ monomer (Santa Cruz Biotechnology, Dallas, TX, USA), functionally active $\beta 3$ (clone CRC54); phosphorylated $\beta 3$ (p-Y759 $\beta 3$ ), importin beta, Lamin B1, GRP78 (Abcam, Cambridge, MA, USA); phospho-PI3K p85 (Tyr458)/p55 
(Tyr199), $\beta$-catenin, vimentin, gapdh, cyclin D1, PARP-1, $\beta$ Tubulin (Cell Signaling, Leiden, The Netherlands); Horseradish peroxidase (HRP) p53 (R\&D systems, Abingdon, UK), PAX8 (Proteintech, Manchester, UK). For IHC and IF of FFPE tissues, $\alpha v \beta 3$ integrin dimer (Abbiotec, San Diego, CA, USA), p53 (Novacastra, Wetzlar, Germany), PAX8 (Cell Marque, Rocklin, CA, USA) and p16 (Ventana, Tucson, Arizona, USA). Secondary HRP-IgG, goat antiRabbit IgG, goat antiMouse IgG (Jackson ImmunoResearch Laboratories, West Grove, PA, USA); Mouse IgGk light chain binding protein-CFL680 (Santa Cruz Biotechnology); DyLight 550 conjugated Donkey antiRabbit antibody (Bethyl Laboratories Inc., Montgomery, TX, USA); IgG- NL493, Donkey antiMouse IgG -NL557 (R\&D systems).

\section{Cell cultures}

HGSOC and FT282/FT237/ FT109 cells were a kind gift from Dr. Ruth Perets (Rambam Medical Center, Haifa, Israel) and $\beta 3 / \beta 1$ HEK293 from Prof. Coll (University of Grenoble, France and CHO-K1 from Prof. Philippe Clézardin (University of Lyon, Lyon, France). OVCAR3, KURAMOCHI, Chinese hamster ovaries (CHO-K1) and HEK293 were grown in full RPMI1640, JHOS4, and FT's in DMEM F-12 and $\beta 3 / \beta 1$ HEK293 in high Glucose DMEM (Biological Industries, Beit Haemek, Israel). STR/ mutation profiling was used for authentication and mycoplasma was screened periodically.

\section{Human tissue samples}

Fresh/archived tissues were collected from nine HGSOC patients at diagnosis undergoing primary debulking surgery and eight nononcological patients undergoing laparotomy/ultrasound guided paracentesis at the Obstetrics and Gynecology Department, Meir Medical Center, Israel. Human tissues were collected with informed consent in compliance with the Institutional Review Board approval, in accordance with the Declaration of Helsinki.

\section{Immunohistochemistry}

Four micron sections were cut from FFPE tissue blocks at the Pathology Department, Meir Medical Center, Israel and stained on a Ventana Benchmark XT automatic stainer (Ventana).

\section{IF confocal microscopy}

Cells were seeded for $24 \mathrm{~h}\left(1 \times 10^{5}\right.$ cells/24 well glass bottom plates). OVCAR3 cells were fixed (4\% PFA), permeabilized $(0.2 \%$ Triton $\times 100)$ and incubated with $10 \mu \mathrm{g} / \mathrm{ml}(1: 100) \alpha v \beta 3$ NL557-labled antibody. JHOS4/ KURAMOCHI were incubated with NL557-labeled $\alpha v \beta 3$ antibody, fixed, permeabilized $(0.2 \%$ Triton $\times 100)$, blocked (1\% BSA) and analyzed for intracellular $\alpha v \beta 3$ by incubation with BP680-labeled $\alpha \mathrm{v} \beta 3$ antibody. For IF staining of FFPE tissues, we performed antigen retrieval (Ventana Benchmark XT automatic stainer), manual blocking (3\% BSA), incubation overnight with $\alpha \mathrm{V} \beta 3$ antibody and staining with secondary antibodies. Nucleus was stained with Hoechst (33342, molecular probes, Eugene, OR, USA). Tissues/cells were analyzed by Leica SP5 confocal microscopy (Leica, Mannheim, German), using $20 \times / 40 \times / 63 \times$ objectives. In all images, a single $0.5 \mu \mathrm{m}$ optical $z$-plane section is presented.

\section{Protein extraction}

Protein fractions were extracted using Fraction PREP TM Cell Fractionation kit (BioVision, Milpitas, CA, USA), Nuclear Extraction Kit (Abcam, Cambridge, MA, USA) or Nuclear Complex Co-IP kit (Active Motif, La Hulpe, Belgium).

\section{Western blot}

Proteins were separated and analyzed as detailed before $^{48}$. Bands were measured by Las3000 imaging system and analyzed by Multigauge v3.0 software (Fujifilm Life Science, Tokyo, Japan).

\section{Flow cytometry}

Absolute cell counts and cell cycle were performed using MACSQuant flow cytometer (Miltenyi Biotec, Bergisch Gladbach, Germany), as detailed before ${ }^{48,49}$.

\section{ImageStream technology}

Amnis ImageStream ${ }^{\circledR \mathrm{X}}$ Mark II multispectral imaging flow cytometer (Amnis Corporation, Seattle, WA, USA) was used. For $\alpha v \beta 3$ membrane expression, $5 \times 10^{5}$ cells were incubated with $10 \mu \mathrm{g} / \mathrm{ml}$ PE-labeled $\alpha \mathrm{v} \beta 3$ dimer antibody, fixed (4\% PFA), permeabilized ( $0.2 \%$ Triton), washed and analyzed for intracellular $\alpha v \beta 3$ by incubation with $10 \mu \mathrm{g} / \mathrm{ml}$ NL493- $\alpha \mathrm{v} \beta 3$ antibody. IgG was used as negative isotype control.

\section{Co-IP}

Nuclear proteins from HEK293ß3 $\left(5 \times 10^{6}\right.$ cells $)$, OVCAR3, JHOS4 and KURAMOCHI $\left(\sim 100 \times 10^{6}\right.$ cells each) were separated and $\alpha v \beta 3$ dimer antibody was used for pull-down using mMACS kit and protein G magnetic beads (Miltenyi Biotec). 3-5 independent biological replicates were conducted. Co-IP from nuclear extracts with beads only, in $\alpha v \beta 3$ negative HEK293 cells $\left(5 \times 10^{6}\right.$ cells), or with isotype IgG, served as negative controls.

\section{NLS- $\beta 3$ vector construction}

Nuclear localization signal sequence (NLS, MDPKKKRKGR ${ }^{50}$ ) was introduced into $\beta 3$ PCEP4 vector at the $c$ terminal end of the mRNA byadding $5^{\prime}$ (SapI) and $3^{\prime}$ (SbfI) sites and custom cloning. The final construct was 
sequenced using PCEP4 EBV-R primer and by Sanger sequencing (outsourced at GENEWIZ, South Plainfield, NJ, USA).

\section{Stable transfections}

OVCAR3 cells were seeded $\left(0.5 \times 10^{6} / 6\right.$ well plates $)$ and transfected a day later with the various vectors $(2.5 \mu \mathrm{g})$ using $7.5 \mu \mathrm{l}$ of TransIT-2x transfection regent (Mirus, Madison, WI, USA). After $48 \mathrm{~h}$, selection antibiotic (Hygromycin B, Merck Millipore) was added at $0.75 \mathrm{mg} /$ $\mathrm{ml}$ to initiate stable cell lines.

\section{Wound healing assay}

IncuCyte $\mathrm{ZOOM}^{\mathrm{TM}}$ real time live cell imaging system (Essen BioScience, MI USA) was used to calculate the relative wound density within the initially-vacant area at each time point.

\section{Soft agar colony assay}

OVCAR3 cells were transfected with PCEP4/ $\beta 3 / \beta 3-\mathrm{NLS}$ vectors, suspended in $0.6 \%$ noble agar (A5431, Sigma Aldrich $)$ in growth medium and plated $\left(2.5 \times 10^{5} / 6\right.$ well plates) onto layer of $0.9 \%$ noble agar in growth medium. Fresh medium was added twice a week for 4 weeks. Plates were stained overnight with $0.05 \%$ crystal violet in $10 \%$ Neutral Buffered Formalin (316-155, Thermo Fisher Scientific), washed and colonies were counted using OpenCFU software. Experiments were repeated twice.

\section{Proteomics analysis}

Nuclear- $\alpha v \beta 3$-eluted proteins were trypsin digested and analyzed independently or as a pool of two extractions using LC-MS/MS on Q Exactive ${ }^{\mathrm{TM}}$ HF-X Mass Spectrometer (Thermo Fisher Scientific, Bremen, Germany). Proteins were identified by Discoverer software version 1.4 against the Human sequence using the search algorithms Sequest (Thermo Fisher Scientific) and Mascot search engines. Identified peptides were filtered with high confidence, top rank, mass accuracy, and a minimum of two peptides. High confidence peptides have passed the $1 \%$ false discovery rate threshold (Estimated false positives in a list of peptides). Semi-quantification was done by calculating the peak area of each peptide, with an average of the three most intense peptides from each protein. Proteins were considered nuclear integrin-associated (designated as unique) by having greater than 4-fold binding from Co-IP with beads only and complete absence in the negative control cells. Keratins, possible contaminants, were filtered out.

\section{Protein enrichment analysis and classification}

Protein classification was performed using http://www. geneontology.org $/^{51}$ powered by Protein Analysis Through Evolutionary Relationships (PANTHER, http:// pantherdb.org) ${ }^{19}$. Protein-protein interaction network was generated using STRING v. 11 (https://string-db.org) with default settings (minimum required interaction score: medium confidence 0.4$)^{18}$ and clustered to a specified Markov clustering inflation parameter of $3^{52}$. Venny diagram tool (http://bioinfogp.cnb.csic.es/tools/venny) was used to compare shared/unique proteins ${ }^{20}$. ClustVis web tool (https://biit.cs.ut.ee/clustvis) was used for multivariate cluster analysis using PCA and heatmap ${ }^{21}$.

\section{Statistical analysis}

Experiments were repeated independently in triplicates and analyzed by two-sided unpaired or paired $t$-test and/ or by ANOVA for multiple comparisons. Significance was determined at $p<0.05$. Results are presented as mean \pm STE.

\section{Acknowledgements \\ The work of Chen Seraya-Bareket was done in partial fulfillment of the requirements for a PhD degree from the Sackler Faculty of Medicine, Tel Aviv University, Israel. We thank Dr. Rosenberg and Dr. Mor-Cohen (Chaim Sheba Medical Center, Tel-Hashomer, Israel), for the 33 -PCEP4 vector. This work was partly supported by the Varda and Boaz Dotan Research Center in Hemato- Oncology, Tel-Aviv University, Israel.}

\section{Author details \\ ${ }^{1}$ Translational Oncology Laboratory, Hematology Institute and Blood Bank, Meir Medical Center, 44821 Kfar-Saba, Israel. ²Department of Human Molecular Genetics and Biochemistry, Sackler Faculty of Medicine, Tel Aviv University, 6997801 Tel Aviv, Israel. ${ }^{3}$ Sackler Faculty of Medicine, Tel Aviv University, Tel Aviv, Israel. ${ }^{4}$ Gynecologic Oncology Division, Department of Obstetrics and Gynecology, Meir Medical Center, 44821 Kfar Saba, Israel. ${ }^{5}$ Department of Pathology, Meir Medical Center, 44821 Kfar Saba, Israel}

\section{Author contributions}

C.S.B. preformed, analyzed and interpreted the experimental data. A.W. assisted in the IHC assays. E.S.M. and S.T.R. performed some of the WB experiments. D.S performed some of the Co-IP extractions. Y.K., N.A., and A.F. assisted in tissue collection. D.K. and E.E. analyzed the IHC slides. O.A.F. designed, analyzed and interpreted the experimental data. C.S.B., M.E., and O.A.F. wrote the manuscript. All authors read and approved the manuscript.

\section{Data availability}

Full proteomics datasets are available from the corresponding author on reasonable request.

\section{Conflict of interest}

The authors declare that they have no conflict of interest.

\section{Publisher's note}

Springer Nature remains neutral with regard to jurisdictional claims in published maps and institutional affiliations.

Supplementary Information accompanies this paper at (https://doi.org/ 10.1038/s41389-020-00254-2).

Received: 27 April 2020 Accepted: 9 July 2020

Published online: 29 July 2020

\footnotetext{
References

1. Jayson, G. C., Kohn, E. C., Kitchener, H. C. \& Ledermann, J. A. Ovarian cancer. Lancet 384, 1376-1388 (2014).
} 
2. Perets, R. \& Drapkin, R. It's totally tubular....riding the new wave of ovarian cancer research. Cancer Res. 76, 10-17 (2016).

3. Wells, A. \& Marti, U. Signalling shortcuts: cell-surface receptors in the nucleus? Nat. Rev. Mol. Cell Biol. 3, 697-702 (2002).

4. Maisel, S. \& Schroeder, J. Wrong place at the wrong time: how retrograde trafficking drives cancer metastasis through receptor mislocalization. J. Cancer Metastasis Treat. 5, 7 (2019).

5. Xia, W. et al. Nuclear expression of epidermal growth factor receptor is a novel prognostic value in patients with ovarian cancer. Mol. Carcinog. 48, 610-617 (2009).

6. Ohishi, Y. et al. Nuclear localization of E-cadherin but not beta-catenin in human ovarian granulosa cell tumours and normal ovarian follicles and ovarian stroma. Histopathology 58, 423-432 (2011).

7. Desgrosellier, J. S. \& Cheresh, D. A. Integrins in cancer: biological implications and therapeutic opportunities. Nat. Rev. Cancer 10, 9-22 (2010).

8. Liapis, H., Adler, L. M., Wick, M. R. \& Rader, J. S. Expression of alpha(v)beta3 integrin is less frequent in ovarian epithelial tumors of low malignant potential in contrast to ovarian carcinomas. Hum. Pathol. 28, 443-449 (1997).

9. Cruet-Hennequart, S. et al. alpha(v) integrins regulate cell proliferation through integrin-linked kinase (ILK) in ovarian cancer cells. Oncogene 22, 1688-1702 (2003).

10. Hapke, S. et al. Ovarian cancer cell proliferation and motility is induced by engagement of integrin alpha(v)beta3Nitronectin interaction. Biol. Chem. 384, 1073-1083 (2003).

11. Cannistra, S. A., Ottensmeier, C., Niloff, J., Orta, B. \& DiCarlo, J. Expression and function of beta 1 and alpha v beta 3 integrins in ovarian cancer. Gynecol. Oncol. 58, 216-225 (1995).

12. Domcke, S., Sinha, R., Levine, D. A., Sander, C. \& Schultz, N. Evaluating cell lines as tumour models by comparison of genomic profiles. Nat. Commun. 4, 2126 (2013).

13. Knowles, L. M. et al. Integrin av $\beta 3$ and fibronectin upregulate Slug in cancer cells to promote clot invasion and metastasis. Cancer Res. 73, 6175-6184 (2013).

14. Reynolds, A. R. et al. Stimulation of tumor growth and angiogenesis by low concentrations of RGD-mimetic integrin inhibitors. Nat. Med. 15, 392 (2009).

15. Lock, J. G. et al. Reticular adhesions are a distinct class of cell-matrix adhesions that mediate attachment during mitosis. Nat. Cell Biol. 20, 1290-1302 (2018).

16. Novak, M. et al. Stathmin 1 and p16INK4A are sensitive adjunct biomarkers for serous tubal intraepithelial carcinoma. Gynecol. Oncol. 139, 104-111 (2015).

17. Deshmukh, L., Meller, N., Alder, N., Byzova, T. \& Vinogradova, O. Tyrosine phosphorylation as a conformational switch a case study of integrin $\beta 3$ cytoplasmic tail. J. Biol. Chem. 286, 40943-40953 (2011).

18. Szklarczyk, D. et al. The STRING database in 2017: quality-controlled protein-protein association networks, made broadly accessible. Nucleic Acids Res. gkw937 (2016).

19. Mi H, Muruganujan A, Huang X, Ebert D, Mills C, Guo X, et al. Protocol Update for large-scale genome and gene function analysis with the PANTHER classification system (v. 14.0). Nature protocols. 14, 703-721 (2019).

20. Oliveros, J. C. Venny. An interactive tool for comparing lists with Venn's diagrams. 2007-2015 (2007).

21. Metsalu, T. \& Vilo, J. ClustVis: a web tool for visualizing clustering of multivariate data using principal component analysis and heatmap. Nucleic Acids Res. 43, W566-W70 (2015).

22. Humphries, J. D., Chastney, M. R., Askari, J. A. \& Humphries, M. J. Signal transduction via integrin adhesion complexes. Curr. Opin. Cell Biol. 56, 14-21 (2019).

23. Bouvard, D., Pouwels, J., De Franceschi, N. \& Ivaska, J. Integrin inactivators: balancing cellular functions in vitro and in vivo. Nat. Rev. Mol. Cell Biol. 14, 430 (2013).

24. Lin, H. Y. et al. Nuclear monomeric integrin alphav in cancer cells is a coactivator regulated by thyroid hormone. Faseb J. 27, 3209-3216 (2013).

25. Liu, S. et al. A small molecule induces integrin $\beta 4$ nuclear translocation and apoptosis selectively in cancer cells with high expression of integrin $\beta 4$. Oncotarget 7, 16282 (2016).
26. Mullany, L. K. et al. Specific TP53 mutants overrepresented in ovarian cancer impact CNV, TP53 activity, responses to nutlin-3a, and cell survival. Neoplasia 17, 789-803 (2015).

27. Shtutman, M. et al. The cyclin D1 gene is a target of the $\beta$-catenin/LEF-1 pathway. Proc. Natl Acad. Sci. 96, 5522-5527 (1999).

28. Lamouille, S., Xu, J. \& Derynck, R. Molecular mechanisms of epithelialmesenchymal transition. Nat. Rev. Mol. Cell Biol. 15, 178-196 (2014).

29. Ghannam-Shahbari, D. et al. PAX8 activates a p53-p21-dependent proproliferative effect in high grade serous ovarian carcinoma. Oncogene 37, 2213-2224 (2018).

30. Mahipal, A. \& Malafa, M. Importins and exportins as therapeutic targets in cancer. Pharmacol. Ther. 164, 135-143 (2016).

31. Hood, J. D. \& Cheresh, D. A. Role of integrins in cell invasion and migration Nat. Rev. Cancer 2, 91-100 (2002).

32. Hood, J. D., Frausto, R., Kiosses, W. B., Schwartz, M. A. \& Cheresh, D. A. Differential av integrin-mediated Ras-ERK signaling during two pathways of angiogenesis. J. cell Biol. 162, 933-943 (2003).

33. Madrazo, E., Conde, A. C. \& Redondo-Muñoz, J. Inside the cell: Integrins as new governors of nuclear alterations? Cancers 9, 82 (2017).

34. Si, L. et al. MicroRNA-27a regulates the proliferation, chemosensitivity and invasion of human ovarian cancer cell lines by targeting Cullin 5. Arch. Biochem. Biophys. 668, 9-15 (2019).

35. Zhao, G. et al. Cullin5 deficiency promotes small-cell lung cancer metastasis by stabilizing integrin ß1. J. Clin. Investig. 129, 972-987 (2019).

36. Teckchandani, A. \& Cooper, J. A. The ubiquitin-proteasome system regulates focal adhesions at the leading edge of migrating cells. Elife 5, e17440 (2016).

37. Muthu, M., Cheriyan, V. T. \& Rishi, A. K. CARP-1/CCAR1: a biphasic regulator of cancer cell growth and apoptosis. Oncotarget 6, 6499 (2015).

38. Sabatier, R. et al. A seven-gene prognostic model for platinum-treated ovarian carcinomas. Br. J. Cancer 105, 304-311 (2011).

39. Kim, J. H. et al. CCAR1, a key regulator of mediator complex recruitment to nuclear receptor transcription complexes. Mol. Cell 31, 510-519 (2008).

40. Dietel, E., Brobeil, A., Gattenlöhner, S. \& Wimmer, M. The importance of the right framework: mitogen-activated protein kinase pathway and the scaffolding protein PTPIP51. Int. J. Mol. Sci. 19, 3282 (2018).

41. Elad, N. et al. The role of integrin-linked kinase in the molecular architecture of focal adhesions. J. Cell Sci. 126, 4099-4107 (2013).

42. Acconcia, F., Barnes, C. J., Singh, R. R., Talukder, A. H. \& Kumar, R. Phosphorylation-dependent regulation of nuclear localization and functions of integrin-linked kinase. Proc. Natl Acad. Sci. 104, 6782-6787 (2007).

43. Savoy, R. M. \& Ghosh, P. M. The dual role of filamin A in cancer: can't live with (too much of) it, can't live without it. Endocrine 20, R341-R56 (2013).

44. Federico, A. et al. Pan-cancer mutational and transcriptional analysis of the integrator complex. Int. J. Mol. Sci. 18, 936 (2017).

45. Ross, J. A. et al. Eukaryotic initiation factor 5B (elF5B) provides a critical cell survival switch to glioblastoma cells via regulation of apoptosis. Cell Death Dis. 10, 1-15 (2019)

46. Linxweiler, M., Schick, B. \& Zimmermann, R. Let's talk about Secs: Sec61, Sec62 and Sec63 in signal transduction, oncology and personalized medicine. Signal Transduct. Target. Ther. 2, 1-10 (2017).

47. Di Ventura, B. \& Kuhlman, B. Go in! Go out! Inducible control of nuclear localization. Curr. Opin. Chem. Biol. 34, 62-71 (2016).

48. Shinderman-Maman, E. et al. The thyroid hormone-alphavbeta3 integrin axis in ovarian cancer: regulation of gene transcription and MAPK-dependent proliferation. Oncogene 35, 1977-1987 (2016).

49. Cohen, K., Ellis, M., Khoury, S., Davis, P. J., Hercbergs, A. \& Ashur-Fabian, O. Thyroid hormone is a MAPK-dependent growth factor for human myeloma cells acting via alphavbeta3 integrin. Mol Cancer Res. 9, 1385-1394 (2011).

50. Kovács, K. A. et al. TORC1 is a calcium-and CAMP-sensitive coincidence detector involved in hippocampal long-term synaptic plasticity. Proc. Natl Acad. Sci. 104, 4700-4705 (2007).

51. Consortium, G. O. Expansion of the Gene Ontology knowledgebase and resources. Nucleic Acids Res. 45, D331-D8 (2016).

52. Brohee, S. \& Van Helden, J. Evaluation of clustering algorithms for protein-protein interaction networks. BMC Bioinform. 7, 488 (2006). 